\title{
The time evolution of the maximal horizontal surface fluid velocity for an irrotational wave approaching breaking
}

\author{
A. Constantin ${ }^{1,2, \dagger}$ \\ ${ }^{1}$ Department of Mathematics, King's College London, Strand, London WC2R 2LS, UK \\ ${ }^{2}$ Faculty of Mathematics, University of Vienna, Oskar-Morgenstern-Platz 1, 1090 Vienna, Austria
}

(Received 15 October 2014; revised 5 February 2015; accepted 16 February 2015; first published online 10 March 2015)

We derive an equation that relates the evolution in time of the maximum of the horizontal fluid velocity at the surface of an irrotational deep-water plunging or spilling breaker to the first component of the pressure gradient at the surface. The approach applies to overhanging wave profiles, up to breaking time.

Key words: surface gravity waves, wave breaking, waves/free-surface flows

\section{Introduction}

A common property of the sea surface in deep-water regions is its capacity to turn over on itself. Starting from swell (a symmetric profile moving in a practically two-dimensional flow, with negligible variations in the horizontal direction parallel to the wave crest, at constant speed and without change of shape), the hallmark of the onset of breaking is a simultaneous slow increase in amplitude and a steepening of the forward face of the wave. With increasing profile asymmetry a rapid growth of the horizontal fluid velocity at the surface is noticeable in the forward surface region beneath the crest. Thus a portion of the forward surface region beneath the crest is projected in the direction of wave propagation as an overhanging incipient jet that subsequently curls over and impacts on the forward face of the wave, cf. Cokelet (1977). In spilling breakers of moderately long wavelength (so that surface tension effects are weak), crest-front faces become very steep and a small jet forms near the crest. It rapidly impacts on the forward face of the wave, thus producing a small air-water mixture (a plume) that grows by spreading downslope, eventually engulfing a large region of the forward face of the wave, cf. Duncan (2001). A more spectacular and powerful type of breaking waves are plunging breakers, where the wave overturning forms a sizeable forward moving sheet of water which plunges deeply down into the water, some distance from the crest, cf. Banner \& Peregrine (1993). In the deep-water regime, corresponding to water sufficiently deep that the surface waves are unaffected by the direct effects of variations in bed topography,

$†$ Email address for correspondence: adrian.constantin@kcl.ac.uk 
these are the only types of breakers; the other two main types (collapsing and surging breakers) can only occur in the context of shoaling in shallow water, see Perlin, Choi \& Tian (2013). With the bottom topography effect ruled out, the onset of breaking for deep-water waves is due to energy inputs from the interaction with other waves or with opposing currents, as well as from wind forcing, cf. Cokelet (1977). This insight into the breaking of deep-water waves is the outcome of the combination of experimental data and numerical simulations, being beyond the reach of analytical theory even if common simplifying assumptions are made. These simplifying assumptions refer to the neglect of the fluid viscosity for large-scale waves, to the disregard of surface tension effects and to the assumption of irrotational two-dimensional flow.

Our aim is to present a theoretical result. Under the simplifying assumptions that are commonly made in the study of breaking waves, specified above, we will derive an exact equation relating the time evolution of the maximum of the horizontal fluid velocity at the surface to the first component of the pressure gradient at the surface. The approach can cope with overturning deep-water wave profiles. We illustrate the relevance of the equation by showing that it ensures that as long as the point of maximum is located on the forward face of the wave, the maximum has to increase with time.

\section{Preliminaries}

To describe the two-dimensional water flows under investigation we choose rectangular coordinates $(x, y)$ with the horizontal $x$-axis in the direction of wave propagation and the $y$-axis vertical. The motion is assumed periodic in the $x$-direction, with wavelength $L$, but will not be periodic in the time variable $t$. The origin is taken to be on the mean surface level. Since we are interested in large-scale surface deep-water waves, it is reasonable, cf. Longuet-Higgins \& Cokelet (1976) and Cokelet (1977), to consider the fluid inviscid, homogeneous and incompressible. The fluid motion is initiated from rest by conservative forces, so that it will be irrotational at all times prior to breaking. In particular, the slow diffusion of vorticity inwards from the boundaries is neglected. This theoretical set-up for the study of breaking was proposed by Longuet-Higgins \& Cokelet (1976), being pursued in Peregrine, Cokelet \& McIver (1980) and in many subsequent numerical investigations. Experimental confirmation of its practical relevance is provided in Melville, Veron \& White (2002).

Let us now present the governing equations. The equation of motion in the bulk of the fluid is Euler's equation

$$
\begin{gathered}
u_{t}+u u_{x}+v u_{y}=-\frac{1}{\rho} P_{x}, \\
v_{t}+u v_{x}+v v_{y}=-\frac{1}{\rho} P_{y}-g,
\end{gathered}
$$

for the velocity field $(u, v)$, where $g$ is the gravitational constant of acceleration, $\rho$ is the (constant) density of water and $P$ is the pressure. In addition, the equation of mass conservation

$$
u_{x}+v_{y}=0
$$

and the constraint of irrotational flow

$$
u_{y}=v_{x}
$$




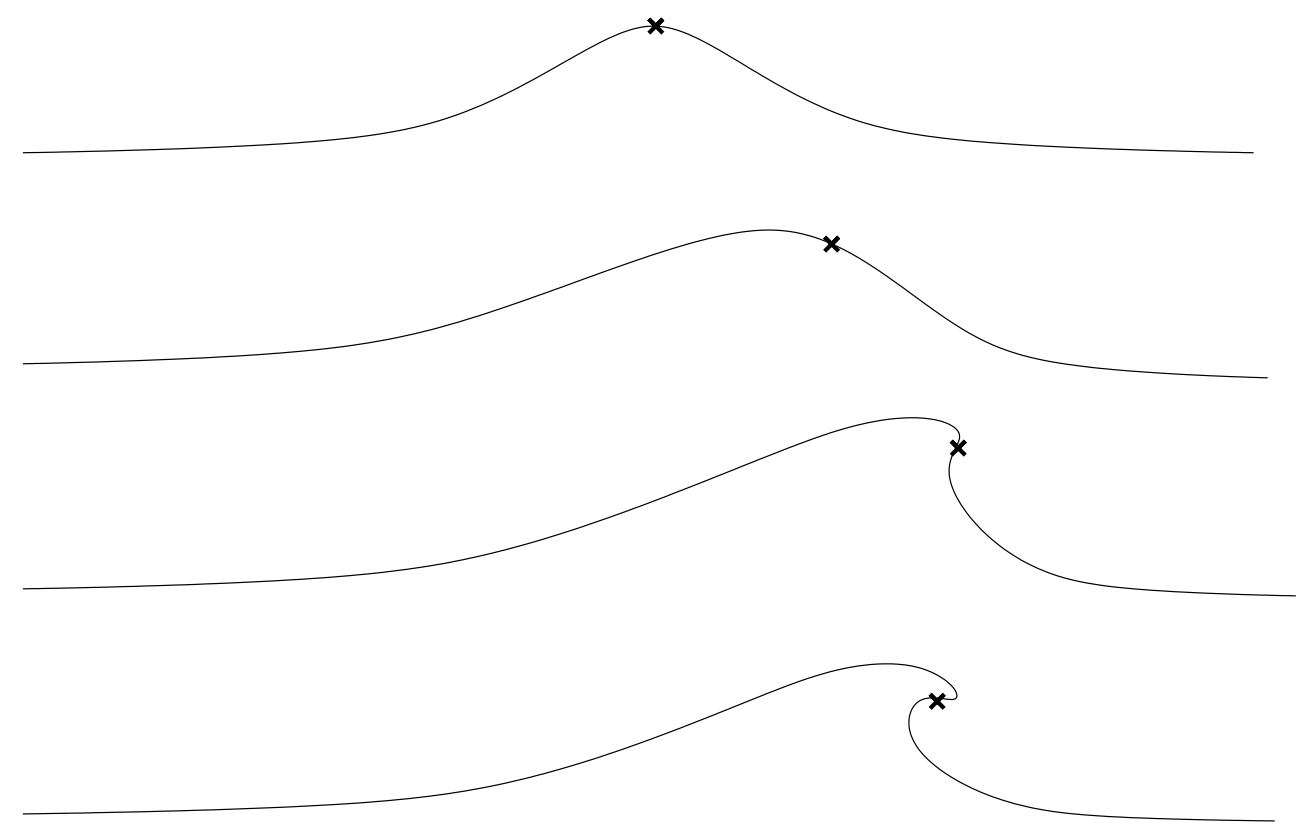

FIGURE 1. Depiction of a typical deformation of a deep-water wave profile at successive times as it steepens and overturns, taking for initial conditions a symmetric travelling wave. The relative size of the overhanging jet distinguishes before breaking between the two breaker types, with small and moderate corresponding to a spilling and plunging breaker, respectively. Experimental data and numerical simulations indicate the location $\mathbf{x}$ of the point where the maximal horizontal fluid velocity at the surface is attained.

should hold within the fluid. Since the dynamics of deep-water waves is unaffected by the bottom topography, for simplicity we consider a flat bed $y=-d$. This assumption is not merely a mathematical convenience since abyssal plains are found in all major sea and ocean basins, covering more than $30 \%$ of the Earth's surface (about the same as all the exposed land combined). On the flat impermeable bed we impose the kinematic boundary condition

$$
v=0 \quad \text { on } y=-d \text {. }
$$

To capture overhanging wave profiles, we represent at a fixed time $t$ the free surface $S(t)$ parametrically as

$$
x=\alpha(s, t), \quad y=\beta(s, t),
$$

where $\alpha$ and $\beta$ are smooth functions with the property that, for every fixed $t$, the map $s \mapsto(\alpha(s, t)-s, \beta(s, t))$ is periodic with wavelength $L$ and such that $\alpha_{s}^{2}(s, t)+$ $\beta_{s}^{2}(s, t)>0$ for every $s \in[0, L]$; the last condition ensures regularity by preventing the existence of singular points. For example, overhanging waves with a profile having per wave period one ascending part and a declining face (common for waves close to breaking, as depicted in figure 1) correspond to $\beta_{s}(s, t)>0$ for $s \in\left(s_{0}(t), s_{+}(t)\right)$ and $\beta_{s}(s, t)<0$ for $s \in\left(s_{+}(t), s_{0}(t)+L\right)$, where $s_{0}(t)$ and $s_{+}(t)$ identify the location of the wave trough and wave crest, respectively. On the free surface the dynamic boundary condition

$$
P(\alpha(s, t), \beta(s, t), t)=P_{a t m},
$$


where $P_{a t m}$ is the constant atmospheric pressure, decouples the motion of the water from that of the air above it. We can ignore the airflow above the water since the density of water is $10^{3}$ times that of air, while surface tension effects are neglected in order to concentrate on the large-scale features of the water waves. Finally, the condition that the free surface is an interface is expressed by requiring that the flow of fluid relative to the free boundary must be tangential to it. The normal to the free surface in space-time being given by the vector product of the tangential vectors $\left(\alpha_{s}, \beta_{s}, 0\right)$ and $\left(\alpha_{t}, \beta_{t}, 1\right)$, we can express the vanishing of the component of fluid velocity normal to the free boundary as the kinematic boundary condition

$$
u \beta_{s}-v \alpha_{s}+\alpha_{s} \beta_{t}-\beta_{s} \alpha_{t}=0 \quad \text { on the free surface } S(t) .
$$

Equations (2.1a)-(2.7) represent the governing equations that we will work with. The existence of smooth solutions as long as the free surface is non-self-intersecting is granted; see the discussion in Constantin (2011). Our aim is to investigate the time evolution of the maximum of the horizontal fluid velocity. Note that we cannot rely on the usual approximate theories for the dynamics of surface waves (the small-amplitude linear theory, the nonlinear shallow-water theory, or variants thereof) since they cease to be valid for overhanging wave profiles.

\section{Main result}

We are interested in the time evolution of the maximum of the horizontal fluid velocity. At any fixed time $t$, this maximum $M(t)$ is attained only along the free surface. Indeed, at a fixed time $t,(x, y) \mapsto u(x, y, t)$ is a harmonic function in the fluid domain $\Omega(t)$, due to (2.2) and (2.3). Since $u$ is not constant, its maximum can only be attained on the boundary. Were $\left(x_{0},-d\right)$ such a location on the flat bed, at time $t_{0}$, this would entail $u_{y}\left(x_{0},-d, t_{0}\right)<0$ by Hopf's maximum principle, see Constantin (2011). But then (2.3) forces $v_{x}\left(x_{0},-d, t_{0}\right)<0$, in contradiction to (2.4). Thus

$$
M(t)=\sup _{(x, y) \in \Omega(t)}\{u(x, y, t)\}=\sup _{s \in[0, L]}\{u(\alpha(s, t), \beta(s, t), t)\}, \quad t \in[0, T),
$$

where $T>0$ is the breaking time. We claim that the function $t \mapsto M(t)$ is absolutely continuous and therefore almost everywhere differentiable, with

$$
M^{\prime}(t)=-\frac{1}{\rho} P_{x}(X(t), t) \quad \text { for almost every } t \in(0, T),
$$

where $X(t)=(x(t), y(t))$ is any location where $u$ attains its maximum.

To prove the validity of (3.2), we first show that $M$ is absolutely continuous with

$$
M^{\prime}(t)=u_{t}(X(t), t)+u_{x}(X(t), t) \alpha_{t}(\xi(t), t)+u_{y}(X(t), t) \beta_{t}(\xi(t), t)
$$

for almost every $t \in(0, T)$, where $\xi(t)$ is such that $X(t)=(\alpha(\xi(t), t), \beta(\xi(t), t))$. Subsequently, we use the fact that

$$
u_{x}(X(t), t) \alpha_{s}(\xi(t), t)+u_{y}(X(t), t) \beta_{s}(\xi(t), t)=0,
$$

since $s=\xi(t)$ is a maximum of the function $s \mapsto u(\alpha(s, t), \beta(s, t), t)$. In combination with (2.7) and (2.1a), we check that this will lead us from (3.3) to (3.2). Before 
providing the details, let us comment on the likelihood of (3.3), drawing attention to some delicate related issues. The existence of $\xi(t)$ being granted, if the function $t \mapsto \xi(t)$ were differentiable, then the function $t \mapsto u(\alpha(\xi(t), t), \beta(\xi(t), t), t)$ could be differentiated and (3.3) would emerge by the chain rule, if we take (3.4) into account. However, this approach cannot be validated since $t \mapsto \xi(t)$ might be multi-valued or, even if it is single-valued, might fail to be continuous. To see this, perform localized uncorrelated time-dependent perturbations near the two peaks at $s_{-}=0$ and $s_{+}=L$ of the periodic function $s \mapsto \cos (2 \pi s / L)$ in the periodicity window $[0, L]$ to set up a scenario in which the location of the maximum in one wave cycle is always at $\xi(t)=s_{+}$for $t>0$, another in which the location is at all times $t>0$ both at $\xi(t)=s_{-}$ and $\xi(t)=s_{+}$(and therefore undetermined), and also a scenario in which the location alternates regularly between the two points $s_{ \pm}$, jumping from one to the other after it becomes undetermined. Nevertheless, the evaluation of $u(\alpha(\cdot, t), \beta(\cdot, t), t)$ at any point $\xi(t)$ where the maximum is attained produces an absolutely continuous function $M(t)$ that satisfies (3.3), a situation reminiscent of that investigated by Constantin \& Escher (1998) in a more abstract setting. To see why this is so, note first that $M(t)$ is locally Lipschitz on $[0, T)$. Indeed, given $\varepsilon>0$, if $t, t^{\prime} \in[0, T-\varepsilon]$ are such that, say, $M(t) \geqslant M\left(t^{\prime}\right)$, then

$$
\begin{aligned}
0 & \leqslant M(t)-M\left(t^{\prime}\right)=u(\alpha(\xi(t), t), \beta(\xi(t), t), t)-u\left(\alpha\left(\xi\left(t^{\prime}\right), t^{\prime}\right), \beta\left(\xi\left(t^{\prime}\right), t^{\prime}\right), t^{\prime}\right) \\
& \leqslant u(\alpha(\xi(t), t), \beta(\xi(t), t), t)-u\left(\alpha\left(\xi(t), t^{\prime}\right), \beta\left(\xi(t), t^{\prime}\right), t^{\prime}\right) \\
& \leqslant \sup _{x \in[0, L]}\left\{\left|u(\alpha(x, t), \beta(x, t), t)-u\left(\alpha\left(x, t^{\prime}\right), \beta\left(x, t^{\prime}\right), t^{\prime}\right)\right|\right\} \leqslant k_{\varepsilon}\left|t-t^{\prime}\right|
\end{aligned}
$$

by the mean-value theorem, with

$$
k_{\varepsilon}=\sup _{\chi \in[0, L], \tau \in[0, T-\varepsilon]}\left\{\left|u_{x}(\mathfrak{X}(\chi, \tau), \tau) \alpha_{t}(\chi, \tau)+u_{y}(\mathfrak{X}(\chi, \tau), \tau) \beta_{t}(\chi, \tau)+u_{t}(\mathfrak{X}(\chi, \tau), \tau)\right|\right\} .
$$

where we have denoted $\mathfrak{X}(\chi, \tau)=(\alpha(\chi, \tau), \beta(\chi, \tau))$. Locally Lipschitz functions being absolutely continuous, they are differentiable almost everywhere, cf. the discussion in Constantin (2011). Since we cannot rely on the chain rule to compute $M^{\prime}(t)$, we argue as follows. Given $t \in(0, T)$ and $h \in(0, T-t)$, we have that $(M(t+h)-M(t)) / h$ equals

$$
\begin{gathered}
\frac{u(\alpha(\xi(t+h), t+h), \beta(\xi(t+h), t+h), t+h)-u(\alpha(\xi(t), t), \beta(\xi(t), t), t)}{h} \\
\geqslant \frac{u(\alpha(\xi(t), t+h), \beta(\xi(t), t+h), t+h)-u(\alpha(\xi(t), t), \beta(\xi(t), t), t)}{h} .
\end{gathered}
$$

In the limit $h \downarrow 0$ the right-hand side of the above estimate converges to the limit

$$
\mathfrak{L}(t)=u_{x}(X(t), t) \alpha_{t}(\xi(t), t)+u_{y}(X(t), t) \beta_{t}(\xi(t), t)+u_{t}(X(t), t) .
$$

Consequently $\liminf _{h \downarrow 0}((M(t+h)-M(t)) / h) \geqslant \mathfrak{L}(t)$. On the other hand, $(M(t)-$ $M(t-h)) / h$ equals

$$
\begin{gathered}
\frac{u(\alpha(\xi(t), t), \beta(\xi(t), t), t)-u(\alpha(\xi(t-h), t-h), \beta(\xi(t-h), t-h), t-h)}{h} \\
\leqslant \frac{u(\alpha(\xi(t), t), \beta(\xi(t), t), t)-u(\alpha(\xi(t), t-h), \beta(\xi(t), t-h), t-h)}{h} .
\end{gathered}
$$


In the limit $h \downarrow 0$ the right-hand side of the above estimate converges to $\mathfrak{L}(t)$, so that $\lim \sup _{h \downarrow 0}((M(t)-M(t-h)) / h) \leqslant \mathfrak{L}(t)$. At an instant $t \in(0, T)$ when $M$ happens to be differentiable we have $M^{\prime}(t)=\liminf _{h \downarrow 0}((M(t+h)-M(t)) / h)=$ $\lim \sup _{h \downarrow 0}((M(t)-M(t-h)) / h)$. This proves the validity of (3.3). To complete the checking of (3.2), let us assume that $M$ is differentiable at the instant $t$ and that $\alpha_{s}(\xi(t), t) \neq 0$. Dividing (3.4) by $\alpha_{s}(\xi(t), t)$ and multiplying the outcome by $\alpha_{t}(\xi(t), t)$, by taking (2.7) into account, we get

$$
\begin{aligned}
& u_{x}(X(t), t) \alpha_{t}(\xi(t), t)+u_{y}(X(t), t) \beta_{t}(\xi(t), t) \\
& \quad=u_{y}(X(t), t) v(X(t), t)-u(X(t), t) u_{y}(X(t), t) \frac{\beta_{s}(\xi(t), t)}{\alpha_{s}(\xi(t), t)} .
\end{aligned}
$$

Using now (3.4), equations (2.1a) and (3.3) lead us to the desired relation (3.2).

\section{Discussion}

The theoretical considerations by Constantin (2006) and Constantin \& Strauss (2010), the numerical simulations performed by Clamond (2012) and the experimental feedback provided by Umeyama (2012) show that for a symmetric travelling wave with a profile that is monotone between successive wave troughs and crests, the maximum $M(t)$ of the horizontal fluid velocity is attained at the crest and does not exceed the propagation speed $c$ of the wave. Kinematic wave breaking criteria based on numerical simulations, laboratory experiments and field observations indicate that with the onset of breaking the maximal horizontal fluid velocity rapidly increases during the overturning process from below $0.85 c$ to almost $30 \%$ greater than $c$, cf. Yasuda, Mutsuda \& Mizutani (1997), Chang \& Liu (1998), Perlin et al. (2013) and references therein. Since this rapid growth in the maximal horizontal fluid velocity is of great interest, let us show that (3.2) enables us to gain some theoretical insight into the dynamical process that occurs with the onset of breaking for a configuration that represents a symmetric travelling wave and is subsequently deformed during the overturning process, up to the breaking point. We can draw the following conclusions, valid also during the development of overhanging, for as long as the wave profile has an ascending part between wave trough and wave crest, and a declining part (the forward face):

(i) Variations of the value of $M(t)$ require the development of asymmetry. As long as symmetry persists, the maximal horizontal fluid velocity is attained at the wave crest.

(ii) Increasing values of $M(t)$ occur only if the point where the horizontal fluid velocity component attains its maximum is located on the forward face of the wave.

For experimental data indicating the location where the maximal horizontal fluid velocity is attained and for the time-evolution of this value, during the deformation of the free surface from a regular travelling wave profile to an overhanging surface wave with a forward plunging jet, we refer to Melville \& Rapp (1988), Yasuda et al. (1997), Mizuguchi (2011); see also figure 1.

To prove the above two claims we rely on (3.2) and on the fact that, at any fixed time prior to breaking, the pressure $P$ is superharmonic in the fluid domain $\Omega(t)$. Indeed, differentiating (2.1a) with respect to the $x$-variable and $(2.1 b)$ with respect to the $y$-variable, adding up the outcomes and taking advantage of (2.2) and (2.3), 
yields $\left(P_{x x}+P_{y y}\right) / \rho=-2 u_{x}^{2}-2 u_{y}^{2} \leqslant 0$ throughout $\Omega(t)$. Superharmonicity ensures that the non-constant function $(x, y) \mapsto P(x, y, t)$ attains its minimum only on the boundary, and at any minimum point its derivative in any direction that exits the fluid domain is strictly negative; see e.g. Constantin (2011) for a discussion of this aspect of elliptic partial differential equations. In particular, we can rule out the flat bed as a possible location for the minimum since (2.4) and (2.1b) ensure that $P_{y}=-\rho g$ on the bed $y=-d$. Due to (2.6), the minimum of $P$ is attained all along the free surface, and the derivative of $P$ in any direction that points outside the fluid, at the free surface, is negative. This argumentation is valid both for profiles that are graphs of a function, of the form $y=\eta(x, t)$, as well as for overhanging profiles that do not self-intersect. To prove Claim (i), assume that the periodic flow pattern remains symmetric, without being necessarily a travelling wave. As long as this is so, if the maximal horizontal fluid velocity is attained at some point $X_{+}(t)$ on the forward face of the wave, the same value must be attained at the symmetric point $X_{-}(t)$ on the free surface. But $X_{-}(t)$ is on the ascending part of the wave profile, and $X_{+}(t)$ on the declining part, so that $P_{x}\left(X_{-}(t), t\right)>0$ and $P_{x}\left(X_{+}(t), t\right)<0$, unless the location is at the wave crest or wave trough. Since on the right-hand side in (3.2) we are allowed to choose any point $X(t)$ where the maximum is attained, the two opposite inequalities cannot be reconciled. Therefore the location is either at the wave crest or at the wave trough. Moreover, initially we start from a symmetric travelling wave, in which case the value of $u$ at the wave trough is smaller than the value at the wave crest. Consequently, the maximum is located at the wave crest, where $P_{x}=0$. Thus $M^{\prime}(t)=0$ almost everywhere in the time interval in which symmetry persists. Knowing that $t \mapsto M(t)$ is absolutely continuous, by integration we conclude that $M(t)$ remains constant. This completes the proof of Claim (i). Claim (ii) is validated by noticing that on the forward face (excluding the wave crest and the wave trough) of the wave $P_{x}<0$, while $P_{x}>0$ on the other face.

\section{Acknowledgements}

Helpful comments and suggestions of the referees on an earlier draft of this paper and the support of the FWF Project I544-N13 'Lagrangian kinematics of water waves' of the Austrian Science Fund are gratefully acknowledged.

\section{REFERENCES}

Banner, M. L. \& Peregrine, D. H. 1993 Wave breaking in deep water. Annu. Rev. Fluid Mech. 25, 373-397.

Chang, K.-A. \& LiU, P. L.-F. 1998 Velocity, acceleration and vorticity under a breaking wave. Phys. Fluids 10, 327.

Clamond, D. 2012 Note on the velocity and related fields of steady irrotational two-dimensional surface gravity waves. Phil. Trans. R. Soc. Lond. A 370, 1572-1586.

COKELET, E. D. 1977 Breaking waves. Nature 267, 769-774.

Constantin, A. 2006 The trajectories of particles in Stokes waves. Invent. Math. 166, 523-535.

Constantin, A. 2011 Nonlinear Water Waves with Applications to Wave-Current Interactions and Tsunamis, CBMS-NSF Reg. Conf. Ser. Appl. Maths, vol. 81. SIAM.

CONSTANTIN, A. \& EsCHER, J. 1998 Wave breaking for nonlinear nonlocal shallow water equations. Acta Mathematica 181, 229-243.

Constantin, A.\& Strauss, W. 2010 Pressure beneath a Stokes wave. Commun. Pure Appl. Maths 63, 533-557.

Duncan, J. H. 2001 Spilling breakers. Annu. Rev. Fluid Mech. 33, 519-547. 
Longuet-Higgins, M. S. \& Cokelet, E. D. 1976 The deformation of steep surface waves on water. Proc. R. Soc. Lond. A 350, 1-26.

Melville, W. K. \& RAPP, R. J. 1988 The surface velocity field in steep and breaking waves. $J$. Fluid Mech. 189, 1-22.

Melville, W. K., Veron, F. \& White, C. J. 2002 The velocity field under breaking waves: coherent structures and turbulence. J. Fluid Mech. 454, 203-233.

Mizuguchi, M. 2011 Experimental study on kinematics and dynamics of wave breaking. Coast. Engng Proc. 20, 589-603.

Peregrine, D. H., Cokelet, E. D. \& McIver, P. 1980 The fluid mechanics of waves approaching breaking. In Proceedings of the 17th International Conference on Coastal Engineering (ed. B. L. Edge), pp. 512-528. ASCE.

Perlin, M., Choi, W. \& Tian, Z. 2013 Breaking waves in deep and intermediate waters. Annu. Rev. Fluid Mech. 45, 115-145.

Umeyama, M. 2012 Eulerian-Lagrangian analysis for particle velocities and trajectories in a pure wave motion using particle image velocimetry. Phil. Trans. R. Soc. Lond. A 370, 1687-1702.

Yasuda, T., Mutsuda, H. \& Mizutani, N. 1997 Kinematics of overturning solitary waves and their relations to breaker types. Coast. Engng 29, 317-346. 